\title{
Effects of Berberine on the Chondrogenic Differentiation of Embryonic Limb Skeletal Progenitors
}

\section{Cristina Duarte-Olivenza Juan Antonio Montero Carlos Ignacio Lorda- Diez (1D}

Departamento de Anatomía y Biología Celular and IDIVAL, Universidad de Cantabria, Santander, 390II, Spain
Correspondence: Carlos Ignacio Lorda-Diez Departamento de Anatomía y Biología Celular and IDIVAL, Universidad de Cantabria, Santander, 390II, Spain Tel +34 94220I570

Fax +34 942201923

Email lordaci@unican.es
Introduction: Berberine (BBR) is an isoquinoline plant alkaloid with demonstrated antiinflammatory, anti-tumor and immunosuppressive pharmacological properties that functions via multiple signaling pathways and epigenetic modulators. Numerous studies have proposed BBR as a promising therapeutic agent for joint cartilage degeneration, and other connective tissue diseases. Purpose and Methods: This work aimed to evaluate the effects of BBR on the growth and differentiation of embryonic skeletal progenitors using the limb mesoderm micromass culture assay.

Results: Our findings show that at difference of its apoptotic influence on a variety of tumor tissues, cell death was not induced in skeletal progenitors by the addition of 12 or $25 \mu \mathrm{M}$ BBR concentration to the culture medium. Morphological and transcriptional analysis revealed dual and opposite effects of BBR treatments on chondrogenesis depending on the stage of differentiation of the cultured progenitors. At early stage of culture, BBR was a potent chondrogenic inhibitor, while chondrogenesis was intensified in treatments at advanced stages of culture. The chondrogenic promoting effect was accompanied by a moderate upregulation of gene markers of prehypertrophic cartilage, including ColXal, alkaline phosphatase Alpl, Runx2, and Indian Hedgehog $I h h$. We further observed a positive transcriptional influence of BBR in the expression of DNA methyltransferase genes, Dnmt1, Dnmt3a and Dnmt $3 b$, suggesting a potential involvement of epigenetic factors in its effects. Conclusion: Our study uncovers a new pharmacological influence of BBR in cartilage differentiation that must be taken into account in designing clinical protocols for its employment in the treatment of cartilage degenerative diseases.

Keywords: chondrogenesis, osteoarthritis, cartilage differentiation, DNA methyltransferases

\section{Introduction}

The degenerative pathology of the locomotor system shows a high prevalence in occidental society due to the aging of the population, to the sedentary lifestyle, and to the practice of highly competitive sports. Joint cartilage, ligaments and tendons are major targets of such degenerative diseases. Regenerative medicine offers great expectations for the treatment of these diseases. However, the development of this therapy requires a detailed knowledge of the mechanisms that regulate the differentiation and functional maintenance of skeletal tissues. Epigenetic regulators are emerging as major factors responsible for these functions. ${ }^{1}$

The embryonic limb provides a useful model for analyzing differentiation of skeletal tissues and for testing new therapeutic approaches. The early developing 
limb is formed by a core of skeletal progenitors of somatopleural origin covered by ectoderm. During the development, progenitors are segregated into a core region that aggregate to form the cartilage templates of the skeleton and a peripheral population that remains undifferentiated under the influence of the ectodermal cover. $^{2}$ The peripheral cell population forms the connective tissues associate with joints and muscles, ${ }^{3}$ or are eliminated by cell death. ${ }^{4}$ Most evidence indicate that the structural diversification of limb skeletal progenitors in vivo is regulated by epigenetic modifications via Wnt/ $\beta$-catenin signaling or by functional regulation of DNA methyl transferases. ${ }^{2,5,6}$ In vitro studies analyzing the chondrogenic differentiation of stem cells revealed important and precocious transcriptional changes in the expression of pro-chondrogenic factors such as Sox9, Sox5, Sox6, and Runx2 via epigenetic modifications. ${ }^{7,8}$ In addition, SOX9 that is a master chondrogenic factor regulates itself the methylation status of downstream chondrogenic markers. ${ }^{9}$ Transcriptional changes regulated by epigenetic modifications are also associated with tendon differentiation and aging, ${ }^{10,11}$ and with the progress of cartilage differentiation. $^{12,13}$

Berberine (BBR) is a botanical alkaloid employed in traditional Chinese medicine that has been characterized as a promising therapeutic agent for numerous degenerative and inflammatory diseases, and also as anti-tumoral treatment for a variety of cancers. ${ }^{14-16}$ Among the pharmacological applications proposed for BBR are skeletal degenerative diseases, including joint cartilage degeneration and osteoarthritis, ${ }^{14-18}$ intervertebral disc degeneration, ${ }^{19,20}$ and abnormal fibrosis. ${ }^{21}$

It is believed that the signaling pathways associated with inflammation are major molecular targets for the pharmacological effects of BBR. ${ }^{22}$ However, there is increasing evidence showing that transcriptional gene regulations via epigenetic modifications sustain, at least in part, its pharmacological effects. ${ }^{23-26}$ It has been reported that BBR ameliorates cartilage degeneration via $\mathrm{Wnt} / \beta$-catenin signal pathway, ${ }^{27}$ and exerts a potent antitumoral effect on multiple myeloma inducing the degradation of UHRF1 via the ubiquitin-dependent proteasome system. ${ }^{25}$ Considering that both $\beta$-catenin signaling and UHRF1 are important regulators of differentiation of limb chondroprogenitors, ${ }^{2,28,29}$ and the proposed beneficial effect of BBR on cartilage degeneration, ${ }^{27}$ the aim of our study was to explore its effect on embryonic limb skeletal progenitors at different stages of differentiation.

\section{Materials and Methods}

\section{Micromass Cultures}

The study was performed in high density cultures of skeletal progenitors (micromass cultures) obtained from the undifferentiated mesoderm of the distal margin of the limb bud ("progress zone") of chick (Rhode Island) embryos at 4.5 day of incubation, equivalent to stages 24 and $25 \mathrm{HH}$. Fertilized eggs were obtained from a commercial farm (Granja Santa Isabel. Cordoba Spain) and embryo extraction was performed at the appropriate stage following the ethical recommendations of the European Communities Council. Embryos were washed in cold PBS and the most distal part of the hindlimb bud was dissected out. The tissue fragments were dissociated to single-cell level by treatment with $0.25 \%$ trypsin and $0.25 \%$ collagenase for 6 and $12 \mathrm{~min}$ respectively. The cellular suspension was then filtered through a $100 \mu \mathrm{m}$ membrane and resuspended in Dulbecco's Modified Eagle Medium (DMEM, Lonza) supplemented with 10\% fetal bovine serum (FBS), 100 units $/ \mathrm{mL}$ penicillin and 100mg/ $\mathrm{mL}$ streptomycin. $10 \mu \mathrm{L}$ drops of cell suspension at a concentration of $2 \times 10^{4}$ cells $/ \mu \mathrm{L}$ were pipetted into each well of a 48 well plate. Cells were allowed to attach for 2 hours and then $200 \mu \mathrm{L}$ of DMEM was added to each well. "Short term cultures" were incubated for 2 days in DMEM with or without $10 \%$ FBS. "Long-term cultures" were incubated for 12 days in DMEM plus $10 \% \mathrm{FBS}$ and $50 \mathrm{mg} / \mathrm{mL}$ of ascorbic acid added to the medium from day 7 of culture. We have previously reported, ${ }^{30}$ that these culture periods corresponds to pre-chondrogenic and pre-hypertrophic stages of cartilage differentiation.

At the end of the initial culture period, both short-term and long-term cultures were cultured for additional $6 \mathrm{hr}$ in a medium containing 12.5 or $25 \mu \mathrm{M}$ Berberine (BBR, Sigma-Aldrich) dissolved in DMSO. Control cultures were maintained for the same period in fresh medium lacking BBR but containing the same amount of DMSO. At the end of this period, cell proliferation and apoptosis were studied by flow cytometry and transcriptional changes were analyzed by Real-time quantitative PCR (qPCR). The phenotypic outcome of control and experimental cultures was analyzed by cartilage specific staining with Alcian Blue or by histology after an additional 3 days of culture in drug-free medium.

\section{Morphological Analysis}

The chondrogenic differentiation of the cultures was analyzed by whole mount staining with $0.5 \%$ Alcian blue at 
pH1.0 of cultures fixed in Kahle's solution. The intensity of Alcian blue staining was quantified by colorimetric analysis at $600 \mathrm{~nm}$ after dye extraction with 6 $\mathrm{M}$ guanidine- $\mathrm{HCl}$ ( $\mathrm{pH}$ 5.8). Each sample was a pool of 4 micromasses. Data were analyzed by ANOVA followed by Bonferroni test for post-hoc comparisons and statistical significance was set at $\mathrm{p}<0.05$.

For histology, micromasses were detached from the wells and embedded in araldite. $1 \mu \mathrm{m}$ thick sections were obtained and stained with Toluidine blue.

Cell senescence was evaluated detecting $\beta$ galactosidase activity at $\mathrm{pH} 6$ (SA $\beta$-gal) after overnight fixation in $4 \%$ glutaraldehyde following the recommendations of Debacq-Chainiaux et al. ${ }^{31}$ The SA $\beta$-gal positive area in the central region of each culture was measured with Image $\mathrm{J}$ default tools and values were analyzed by ANOVA followed by Bonferroni test.

Apoptosis was evaluated by the terminal deoxynucleotidyl transferase-mediated dUTP-TRIC nick end labeling (TUNEL) assay using the in situ cell death detection kit (Roche).

\section{Flow Cytometry}

Control and BBR-treated cell cultures were dissociated with trypsin EDTA (Lonza). For propidium iodide (PI) staining, cells were washed with PBS, and fixed in $90 \%$ ethanol and stained overnight at $4^{\circ} \mathrm{C}$ with $0.1 \mathrm{mg} / \mathrm{mL}$ PI with $0.1 \%$ sodium citrate and $0.01 \%$ Triton $\mathrm{X}-100$. The cell suspensions were subjected to flow cytometry in a Cytoflex (Beckman Coulter) with Cytexpert software for data collection and analysis. One million cells were used in each test. The gating strategy was made by deselecting impurities (debris) using an SSC-A/IP-PE dot plot and then selecting the populations of apoptotic and living cells in a logarithmic scale IP-PE histogram (see Supplementary Figure 1). Data were analyzed by ANOVA followed by Bonferroni test for post-hoc comparisons. Statistical significance was set at $\mathrm{p}<0.05$.

\section{Real-Time Quantitative PCR (qPCR) for Gene Expression Analysis}

For transcriptional analysis we selected a panel of genes including: master transcription factors of distinct connective tissues (Sox9, Scleraxis, Runx2, Tgif1, Ihh and Alpl); epigenetic regulators (Uhrf1, Uhrf2, Dnmt1, Dnmt3a, Dnmt $3 b$ and Tet3); cell senescence ( $p 16, p 21, p 53, p 63$ and $p 73$ ); Tumor suppressor genes (Btg1 and Btg2); apoptotic markers (Bakl); TGF $\beta$ ligands (Tgf $\beta 2$ and $T g f \beta 3)$; and a variety of extracellular matrix constituents and reg-

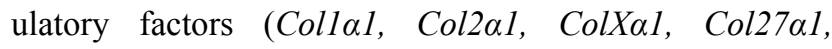
Aggrecan, Decorin, the matricellular proteins $C c n 1$ and Ccn3, and the metalloprotease Mmp2).

Total RNA from Control or BBR-treated cultures was extracted using the NucleoSpin RNA Kit (MachereyNagel). RNA samples were quantified using a spectrophotometer ND-1000 (Nanodrop Technologies). First-strand cDNA was synthesized by RT-PCR using the RevertAid RT Kit (Thermo Scientific). The cDNA concentration measured in a spectrophotometer ND-1000 was adjusted to $0.5 \mu \mathrm{g} / \mu \mathrm{L}$. SYBR Green qPCRs were performed using the StepOne system (Life Technologies), with automation attachment, and the $\mathrm{SYBR}^{\circledR}$ Select Master Mix (Applied Biosystems). Gapdh had no significant variation in expression across the sample set and therefore was chosen as the normalizer in our experiments. Mean values for fold changes were calculated for each gene. Each value in this work represents the mean \pm SD of at least five independent samples obtained under the same conditions from at least 3 distinct experiments. Samples consisted of four micromass cultures. Student's $t$-test was employed to analyze the statistical significance when control and experimental sample values differed more than 1.5 folds. Primers for qPCR are included in Supplementary Table S1.

\section{Results}

The micromass culture assay is a precursor of what we now term organoid cultures. ${ }^{32,33}$ Dissociated limb undifferentiated skeletal progenitors cultured at high density in serumfree minimum essential medium or in medium containing $10 \%$ fetal bovine serum recapitulates the events leading to the formation of the skeletal primordia except for the absence of the specific morphological shape of cartilages in vivo. ${ }^{34,35}$ After two days of culture, cells aggregate forming small SOX9 and Alcian blue positive nodules. Cells in the perinodular regions maintain a fibroblastic appearance. Consistent with this differentiation pattern, both Scleraxis and Sox 9 genes are robustly expressed at these stages. Scleraxis is a marker of connective tissues that in vivo surround the differentiating cartilaginous skeleton, including tendons, joint tissues, and aponeurosis. ${ }^{36}$ In subsequent days of culture, the chondrogenic nodules expand both in number and in size at expense of reducing the amount of peripheral fibroblastic tissue. The progression of chondrogenesis is intense in cultures containing FBS and more limited when cultures are grown only in minimum essential 
medium. After 10 days of culture in medium lacking FBS, and some days earlier in cultures containing FBS, cartilage specifically stainable by Alcian blue is the predominant tissue in the culture. In a previous study we observed that cultures of 2 and 12 days represent two distinct stages of cartilage differentiation. 2 days cultures contain prechondrogenic cells in course of aggregation while 12 days cultures contain maturing cartilage in a prehypertrophic stage of differentiation. ${ }^{30}$ Consistent with these data, expression of Collagen type 2 alphal (Col2 $\alpha 1$ ) and Aggrecan genes, that are master markers of chondrodifferentiation increased by 5 and 7 folds respectively between day 2 and day 12 of culture (Fig. Supplementary 1). Considering these stages of cartilage differentiation, we performed treatments at early (short-term) and late stages (long-term) of culture to monitor the differences in the influence of BBR over the immature chondroprogenitors versus differentiating chondrocytes. We analyzed the outcome and differentiation of the cultures and the transcriptional regulation that precede differences in chondrodifferentiation.

\section{BBR Decreased Initial Stages of Chondrogenesis Favoring Fibroblastic Differentiation of Progenitors}

Although the differentiation of the micromass cultures progressed much quicker in the presence of FBS than in its absence, in both cases, a 6hr treatment of BBR at the end of the second day of culture caused an intense inhibition of chondrogenesis detectable 3 days later. As shown in Figure 1, after three days of additional culture in BBRfree medium, the number, size, and staining intensity of the Alcian blue-positive nodules were reduced in BBRtreated micromasses (Figure 1A and C). Notably, the perinodular fibroblastic tissue increased in a dose-dependent fashion in parallel with the reduction of the size of the Alcian blue positive nodules (Figure 1B, D, F-H). In tissue sections of control micromasses, cartilage nodules exhibit a well structural organization and are separated of each other by a reduced amount of internodular tissue (Figure $1 \mathrm{~B}$ and $\mathrm{F}$ ). In contrast, chondrogenic regions of BBR-treated cultures appeared less organized with zones having a rather fibrocartilaginous appearance with rounded chondrocytes rich in extracellular matrix intermingled with regions of dark cells lacking the morphological appearance of chondrocytes (Figure 1G-H). Quantification of the Alcian blue extracted by guanidine- $\mathrm{HCl}$ showed a $50 \%$ fall of chondrogenesis in cultures treated with $12.5 \mu \mathrm{M}$
BBR (hereafter, "low-dose" of BBR) and 70\% fall in $25 \mu \mathrm{M}$ BBR treatments (hereafter "high-dose" of BBR) (Figure 1I).

Changes in cell proliferation, cell senescence and cell death together with transcriptional modifications preceding the different outcome of the treated cultures were analyzed at the end of the $6 \mathrm{hr}$ period of BBR treatment in cultures growing in DMEM without FBS. Not statistically significant changes in cell cycle, cell senescence or cell death between control and treated cultures were appreciated in this period, even in high-dose BBR treatments (Figure 1J, $\mathrm{K}$, and Fig. Supplementary 2).

As shown in Table 1, we observed mild but significant transcriptional changes in genes associated with for cell differentiation, cell senescence, cell death, and epigenetic regulation. Independent of the doses selected, $\operatorname{Tg} f \beta 2$ and Scleraxis were up regulated. Other genes belonging to the TGF $\beta$ pathway involved in fibrogenesis or the transcription factor Runx2, which play a role at initial and at advanced stages of chondrogenic differentiation, ${ }^{35,37}$ were not regulated. $p 21$ and $p 53$ genes, but not p16, that are developmental senescence central factors, ${ }^{38}$ were moderately up-regulated although the pattern of SA $\beta$-gal staining appeared only moderately intensified without reaching statistically significance (Fig. Supplementary 2). Tumor suppressor genes and the pro-apoptotic factor Bakl were neither regulated at significant levels. Among the epigenetic regulators, Dnmt1, 3a, and $3 b$ were all significantly up regulated, but other epigenetic modulators were not. Extracellular matrix factors were not significantly regulated.

\section{BBR Promotes Cartilage Maturation at Advanced Stages of Culture}

Cartilage is the predominant component of micromasses cultured for 12 days. At this stage, the cultures were widely positive for Alcian blue staining showing nodules of intensified staining (Figure 2A). At difference of treatments at earlier stages of culture (short-term), chondrogenesis appeared strongly intensified in cultures treated with low-dose BBR, (Figure 2B) and in a less intense fashion after treatment at high-dose of BBR (Figure 2C). In tissue sections, control and the low-dose treated experimental cultures showed similar appearance (Figure 2D-E). The high dose-dose treated cultures showed a slight prehypertrophic morphology (Figure 2F). Quantification of chondrogenesis after guanidine- $\mathrm{HCl}$ extraction of Alcian blue 


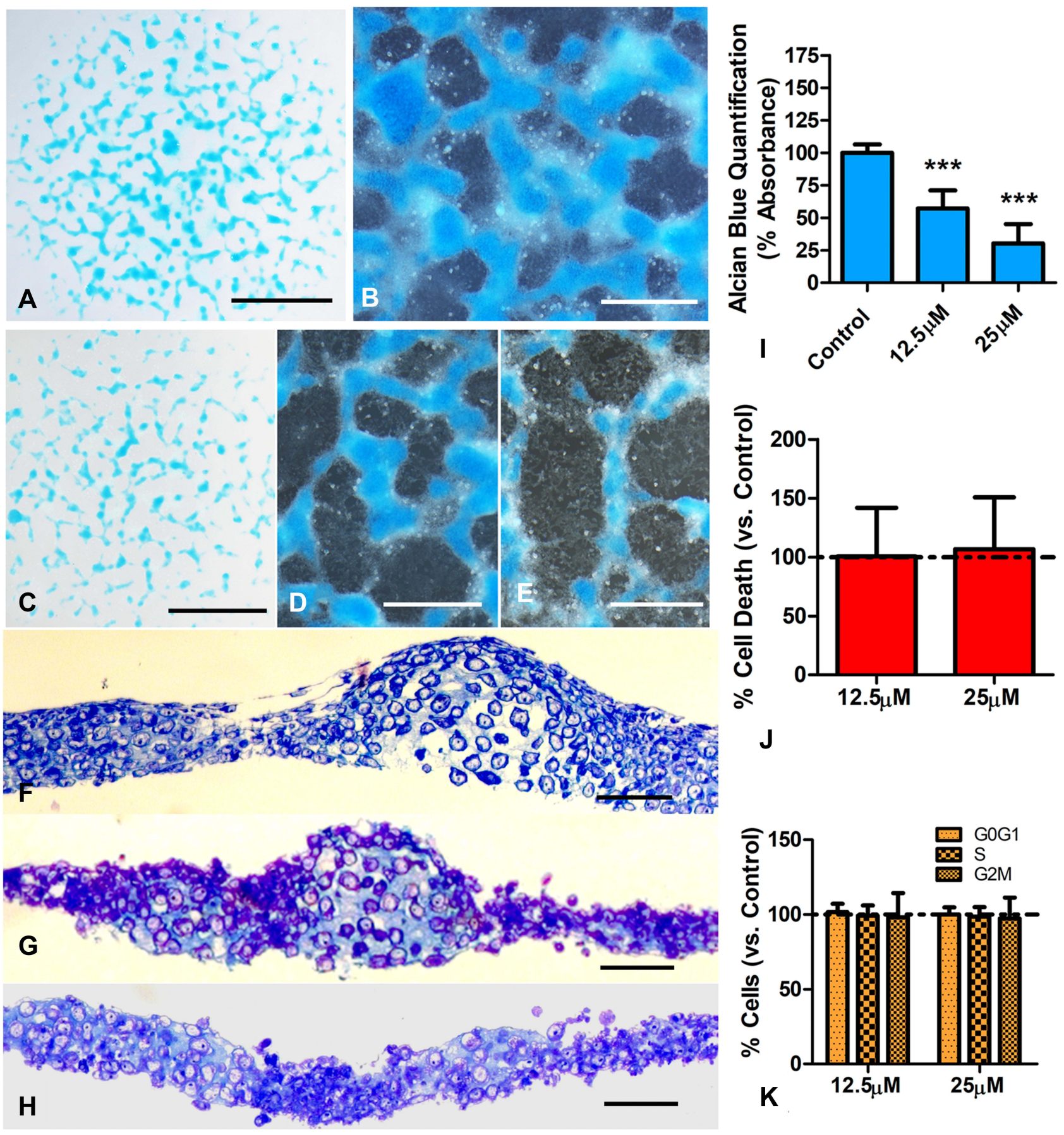

Figure I Effects of BBR in 2-day cultures of limb skeletal progenitors. (A and B) Low magnification (A) and detailed view (B) of 5 days old micromass cultures after Alcian blue cartilage staining. (C and D) low magnification (C) and detailed (D) views of similar cultures to that shown in (A) but subjected to I2.5 $\mu$ M BBR for a transitory 6 hr period at the end of day 2 . Note the intense decrease in Alcian blue positive cartilage nodules. (E) culture treated as in (D), but employing $25 \mu M$ BBR. Note the reduction in size and density of Alcian blue positive nodules at expenses of increasing regions occupied by cells negative for Alcian blue staining. (F-H) semithin sections of control $(\mathbf{F})$ and experimental cultures treated with $12.5 \mu \mathrm{M}$ BBR $(\mathbf{G})$ and $25 \mu \mathrm{M}$ BBR $(\mathbf{H})$ to show the precise structural organization of the differentiating cartilage in the control culture (F) that contrast with a dose-dependent disorganized appearance of cultures subjected to $12.5 \mu \mathrm{M}$ BBR (G) or $25 \mu \mathrm{M}$ BBR $(\mathbf{H})$. Note the diffuse transition of the cartilaginous tissue with the dark cells lacking the cellular an extracellular characteristics of differentiating cartilage in the treated micromasses (G and $\mathbf{H})$. (I) quantitative analysis of Alcian blue dye in control and BBR treated cultures, after guanidine- $\mathrm{HCl}$ extraction $(n=7)$. $(\mathbf{J})$ graphic representation of the rate of cell death evaluated by flow cytometry in short term cultures treated for $6 \mathrm{hr}$ with BBR versus untreated control cultures (represented by the dotted line). (K) graphic representation comparing the proportion of cells at the different stages of the cell cycle between treated and control cultures. Scale bars in $(\mathbf{A}$ and $\mathbf{C})=1 \mathrm{~mm}$; Scale bars in $(\mathbf{B}, \mathbf{D}$ and $\mathbf{E})=400 \mu \mathrm{m}$; scale bars in $(\mathbf{F}-\mathbf{H})=40 \mu \mathrm{m}$. Graphs in $(\mathbf{I}-\mathbf{K})$ represent the Mean \pm SD. The dotted line represents values in control cultures. Differences were analyzed using ANOVA analysis. $* * * \mathrm{p}<0.001$. 
Table I Transcriptional Gene Regulation in Micromass Cultures Treated with BBR for $6 \mathrm{hr}$ at Day 2 Compared with Control Untreated Micromass Cultures

\begin{tabular}{|c|c|c|}
\hline \multicolumn{3}{|c|}{$2 \mathrm{~d}+6 \mathrm{~h}$ BBR } \\
\hline Gene & $12.5 \mu \mathrm{M}$ & $25 \mu M$ \\
\hline \multicolumn{3}{|c|}{ Differentiation } \\
\hline Sox9 & $1.35 \pm 0.23$ & $1.33 \pm 0.21$ \\
\hline Scleraxis & $1.53 \pm 0.39 *$ & $1.93 \pm 0.52 * * *$ \\
\hline Runx2 & $0.97 \pm 0.32$ & $0.70 \pm 0.19$ \\
\hline Tgifl & $1.47 \pm 0.29$ & $1.24 \pm 0.55$ \\
\hline \multicolumn{3}{|c|}{ Apoptosis and Cell Cycle Regulation } \\
\hline p/6 & $1.22 \pm 0.39$ & $1.34 \pm 0.40$ \\
\hline p2I & $1.80 \pm 0.38 * * *$ & $5.04 \pm 1.60 * * *$ \\
\hline p53 & $1.71 \pm 0.62 * *$ & $2.34 \pm 0.8 I *$ \\
\hline p63 & $0.99 \pm 0.30$ & $0.95 \pm 0.32$ \\
\hline p73 & $0.92 \pm 0.41$ & $0.77 \pm 0.33$ \\
\hline Btgl & $1.12 \pm 0.26$ & $1.21 \pm 0.17$ \\
\hline Btg2 & $1.07 \pm 0.26$ & $1.07 \pm 0.28$ \\
\hline Bakl & $1.49 \pm 0.51$ & $1.47 \pm 0.54$ \\
\hline \multicolumn{3}{|c|}{ TGF $\beta$ signaling } \\
\hline $\operatorname{Tgf} \beta 2$ & $1.52 \pm 0.33 * * *$ & $1.79 \pm 0.67 * * *$ \\
\hline $\operatorname{Tgf} \beta 3$ & $1.18 \pm 0.22$ & $1.39 \pm 0.21$ \\
\hline \multicolumn{3}{|c|}{ Epigenetic modulators } \\
\hline Uhrfl & $0.89 \pm 0.22$ & $0.94 \pm 0.39$ \\
\hline Uhrf2 & $1.29 \pm 0.23$ & $1.25 \pm 0.33$ \\
\hline Dnmtl & $1.54 \pm 0.27 * * *$ & $1.52 \pm 0.22 * * *$ \\
\hline Dnmt3a & $1.54 \pm 0.29 * * *$ & $1.5 \mathrm{I} \pm 0.19 * * *$ \\
\hline Dnmt3b & $1.51 \pm 0.32 * *$ & $1.66 \pm 0.43 * * *$ \\
\hline Tet3 & $1.15 \pm 0.60$ & $1.32 \pm 0.68$ \\
\hline \multicolumn{3}{|c|}{ ECM components } \\
\hline Conl & $1.16 \pm 0.17$ & $1.32 \pm 0.04$ \\
\hline Ccn3 & $0.83 \pm 0.19$ & $0.60 \pm 0.26$ \\
\hline Coll $\alpha I$ & $1.04 \pm 0.27$ & $1.06 \pm 0.52$ \\
\hline Col2al & $1.32 \pm 0.30$ & $1.41 \pm 0.23$ \\
\hline Col27 $\alpha I$ & $1.32 \pm 0.32$ & $1.32 \pm 0.25$ \\
\hline Mmp2 & $0.87 \pm 0.23$ & $0.82 \pm 0.25$ \\
\hline Decorin & $1.19 \pm 0.32$ & $1.26 \pm 0.13$ \\
\hline Aggrecan & $1.24 \pm 0.44$ & $1.23 \pm 0.19$ \\
\hline
\end{tabular}

Notes: P-values marked with bold text indicate statistically significant differences between the groups: ${ }^{*} p<0.05$; ${ }^{*} p<0.01$; ${ }^{* * *} p<0.00$ I.

confirmed and $50 \%$ of increased staining in cultures subjected to low-dose of BBR (Figure 2G). The intensification of staining after high-dose BBR treatment was less intense reaching only a $25 \%$ (Figure $2 \mathrm{G}$ ).

As observed in earlier stages of culture, no significant changes in cell death were appreciated after either low- or high-dose BBR treatments (Figure 2H). At difference of treatments at early stage of culture, a moderated slowing at phase $\mathrm{S}$ of the cell cycle, that not reached statistically significance, was appreciated after low-dose BBR treatments (Figure 2I). We choose low-dose treatments for analyzing transcriptional changes (Table 2). Scleraxis, $\operatorname{Tg} f \beta 2, \operatorname{Tg} f \beta 3$ and $\operatorname{Sox} 9$ were all up regulated. Markers of cartilage hypertrophic maturation, ${ }^{39,40}$ including Collagen type X (ColXa1), Runx2, Ihh and alkaline phosphatase gene $(A l p l)$, appeared also positively regulated. The epigenetic marker Dnmt3b, but not other epigenetic regulators, appeared up regulated. Finally, senescence $(p 16, p 21)$ apoptotic (Bakl) and the tumor suppressor Btgl were also upregulated. Detection of SA $\beta$-gal activity appeared moderately intensified after BBR treatments, but without reaching statistically significant differences (Fig. Supplementary 2).

\section{Discussion}

The embryonic limb mesodermal micromass culture assay is a powerful model for analyzing drugs of potential influence on the differentiation and maintenance of skeletal tissues. Limb skeletal progenitors are originated from the somatopleura and have potential to generate all the connective tissues of the limb including, cartilage, tendons, ligaments, fasciae, joint capsules and intramuscular connective tissue. ${ }^{3,41}$ Furthermore, in the absence of appropriate differentiation signals, skeletal progenitors are eliminated by senescence and massive cell death. ${ }^{4}$ Importantly, the diversification of progenitors to form specialized cartilages, fibrous (dense) connective tissue, or being eliminated by cell death appears to be associated with epigenetic changes regulated by local signaling cascades. ${ }^{2,6,13,42}$

BBR has been characterized as a chondroprotective drug that ameliorates cartilage degeneration and joint rigidity in experimental models of osteoarthritis. ${ }^{43-45}$ These findings together with other experimental approaches proposed that BBR could be effective for osteoarthritis treatment, ${ }^{17,22,27,46,47}$ intervertebral disc degeneration, ${ }^{20}$ and pathological fibrotic processes. $^{9}$ Abundant evidence indicates that the anti-inflammatory effect of BBR relays in the regulation of signaling pathways associated with inflammation. ${ }^{15,22}$ Cell death and cell cycle arrest of activated rheumatoid arthritis fibroblast-like synoviocytes in combination with an antiapoptotic influence on chondrocytes were proposed to 

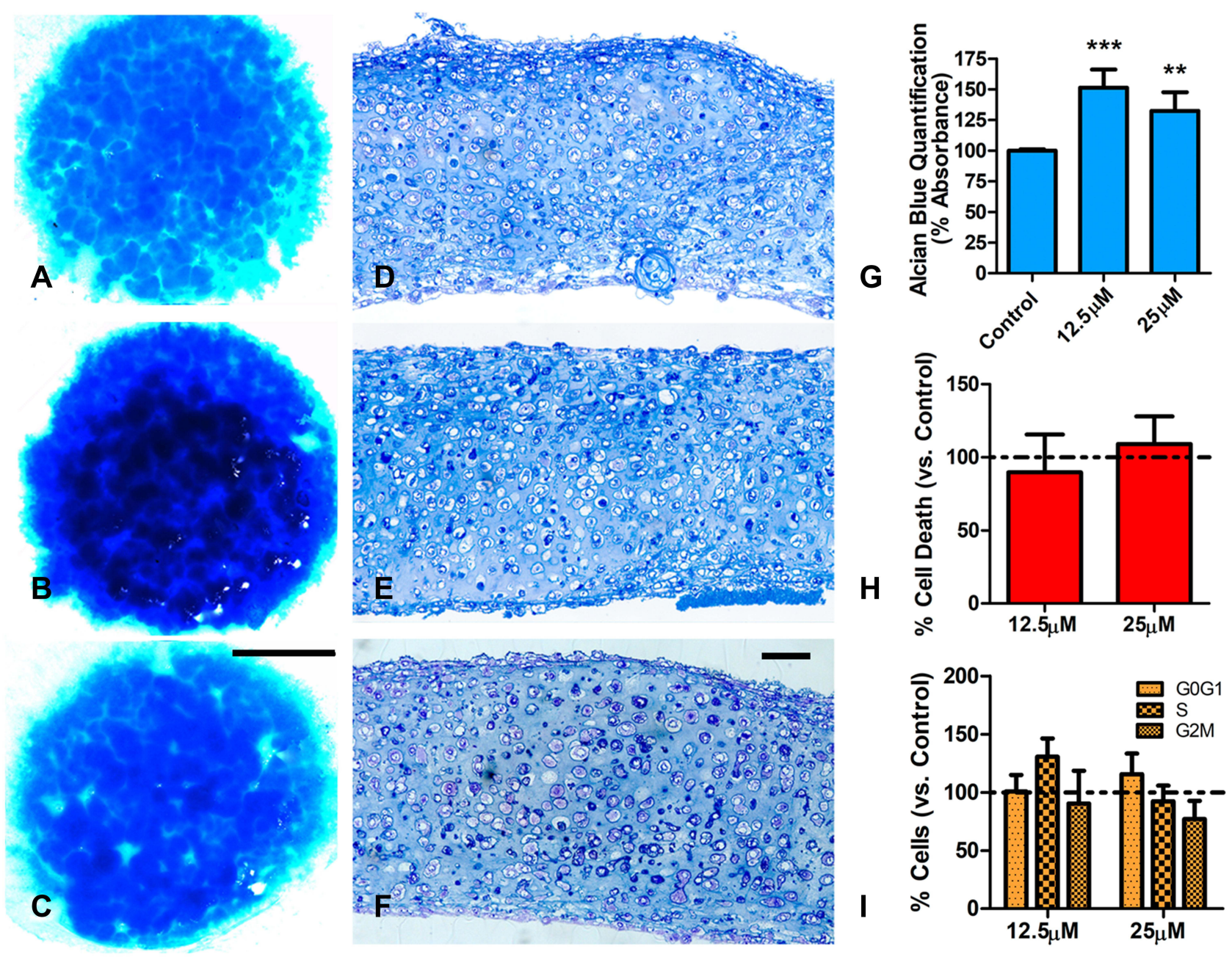

Figure 2 Effects of BBR in 12 days cultures of limb skeletal progenitors. (A-C) are low magnification views of 15 days old cultures stained with Alcian blue. (A) is a control

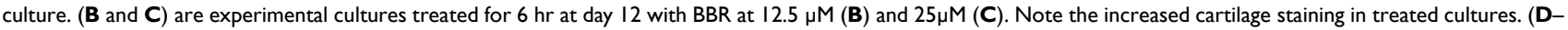
F) semithin sections of control (D) and experimental cultures treated with $12.5 \mu$ M BBR (E) and $25 \mu M$ BBR (F) showing a mild increased prehypertrophic appearance in cartilage subjected to high-dose treatment $(\mathbf{F})$. $(\mathbf{G})$ quantitative analysis of Alcian blue dye in control and $\mathrm{BBR}$ treated cultures, after guanidine- $\mathrm{HCl}$ extraction $(\mathrm{n}=5)$. $(\mathbf{H})$ graphic representation of the rate of cell death evaluated by flow cytometry in long-term cultures treated for 6 hr with BBR versus untreated control cultures (represented by the dotted line). (I) graphic representation comparing the proportion of cells at the different stages of the cell cycle between treated and control cultures. Scale bar for $(\mathbf{A}-\mathbf{C})=I \mathrm{~mm}$; Scale bar for $(\mathbf{D}-\mathbf{F})=30 \mu \mathrm{m}$. Graphs in $(\mathbf{G}-\mathbf{I})$ represent the Mean \pm SD. The dotted line represents values in control cultures. Differences were analyzed using ANOVA analysis. ${ }^{* *} p<0.01$; ${ }^{* * *} p<0.001$.

contribute also to the pharmacological effects of BBR on joint cartilage. ${ }^{44,48}$ Remarkably, the induction of cell cycle arrest and apoptosis constitutes the basis for the antitumoral pharmacological application of BBR in a variety of tumors. ${ }^{15}$ These pharmacological effects have been associated with transcriptional regulation of the proapoptotic and tumor suppressor genes, such as p53, p21, or Bax via inducing epigenetic modifications. ${ }^{15,24,48}$ These modifications included changes in DNA methylation, ${ }^{23}$ inhibition of histone deacetylases binding, ${ }^{24}$ inhibition of the epigenetic regulatory factor $U h r f 1,{ }^{25}$ and transcriptional up-regulation of demethylation (TET) enzymes. ${ }^{26}$
Three major findings concerning the pharmacological properties of BBR can be deduced from our results: 1) firstly, at difference of the apoptotic influence of BBR on a variety of tumoral tissues, no changes in cell death were detected in the micromass culture assay; 2) secondly, in concert with previous studies performed on a variety of normal or pathological tissues, ${ }^{43}$ we found that transcriptional and phenotypic changes of treated cultures caused by BBR administration are associated with the stage of differentiation of cultured progenitors; and, 3), treatments with low doses of BBR performed in cultures at stages of cartilage maturation increased the chondrogenic process. 
Table 2 Transcriptional Gene Regulation in Micromass Cultures Treated with BBR for $6 \mathrm{hr}$ at Day 12 Compared with Control Untreated Micromass Cultures

\begin{tabular}{|c|c|}
\hline \multicolumn{2}{|c|}{ I 2d+6h BBR } \\
\hline Gene & $12.5 \mu \mathrm{M}$ \\
\hline \multicolumn{2}{|c|}{ Differentiation } \\
\hline Sox9 & $2.22 \pm 0.47 *$ \\
\hline Scleraxis & $2.21 \pm 0.96 *$ \\
\hline Runx2 & $1.87 \pm 0.56 * * *$ \\
\hline Tgifl & $1.44 \pm 0.5 \mathrm{I}$ \\
\hline Ihh & $2.8 \mathrm{I} \pm \mathrm{I} .35 * *$ \\
\hline$A \mid p l$ & $1.87 \pm 0.48 * * *$ \\
\hline \multicolumn{2}{|c|}{ Apoptosis and Cell Cycle Regulation } \\
\hline p/6 & $2.32 \pm 0.97 *$ \\
\hline p2I & $1.76 \pm 0.66 * *$ \\
\hline p53 & $1.35 \pm 0.82$ \\
\hline p63 & $1.41 \pm 0.93$ \\
\hline p73 & $1.40 \pm 0.65$ \\
\hline Btgl & $1.58 \pm 0.45 * *$ \\
\hline Btg2 & $1.35 \pm 0.70$ \\
\hline Bakl & $1.68 \pm 0.25 * *$ \\
\hline \multicolumn{2}{|c|}{ TGF $\beta$ signaling } \\
\hline $\operatorname{Tg} \beta 2$ & $1.80 \pm 0.76 *$ \\
\hline $\operatorname{Tg} \beta 3$ & $1.98 \pm 0.86 * *$ \\
\hline \multicolumn{2}{|c|}{ Epigenetic modulators } \\
\hline Uhrfl & $1.14 \pm 0.36$ \\
\hline Uhrf2 & $1.29 \pm 0.45$ \\
\hline Dnmtl & $1.14 \pm 0.39$ \\
\hline Dnmt3a & $1.19 \pm 0.52$ \\
\hline Dnmt3b & $2.06 \pm 1.12 *$ \\
\hline Tet3 & $1.21 \pm 0.62$ \\
\hline \multicolumn{2}{|c|}{ ECM components } \\
\hline Ccnl & $1.51 \pm 0.53 *$ \\
\hline Cen3 & $1.14 \pm 0.52$ \\
\hline Collal & $1.29 \pm 0.30$ \\
\hline Col2al & $1.26 \pm 0.59$ \\
\hline ColXal & $1.57 \pm 0.59 *$ \\
\hline Col27 $\alpha I$ & $1.33 \pm 0.45$ \\
\hline Mmp2 & $1.33 \pm 0.39$ \\
\hline Decorin & $1.02 \pm 0.21$ \\
\hline Aggrecan & $1.07 \pm 0.59$ \\
\hline
\end{tabular}

Notes: P-values marked with bold text indicate statistically significant differences between the groups: ${ }^{*} p<0.05 ;{ }^{* *} p<0.01 ; * * * p 0.001$.

It has been found that the pro-apoptotic and antiproliferative effects of BBR in breast cancer cells are not observed in non-malignant breast epithelial cells. ${ }^{49}$ The absence of increased cell death in our experimental assay is consistent with the reported chondroprotective influence of $\mathrm{BBR},{ }^{44}$ and supports the specificity of the dying influence of BBR on different cell lineages. As reported in different experimental studies, ${ }^{22,48,50}$ we have observed a mild, but significant, transcriptional influence of BBR on genes associated with cell death and developmental cell senescence, ${ }^{51,52}$ including $p 21$ and Bak1, p53 and the tumor suppressor gene Btg1. This moderate gene up-regulation in our in vitro assay appeared insufficient to induce apoptosis or increased SA $\beta$-gal activity in early or late cultures at the end of the $6 \mathrm{hr}$ period of treatment.

The increased chondrogenesis observed after treatments of cultures at advanced stages of cartilage differentiation might be important when considering the therapeutic influence of BBR on joint cartilage degeneration. $^{22}$ In our experimental model, BBR at initial stages of culture inhibited chondrogenesis, up-regulating pro-fibrogenic genes (Tgf $\beta 2$ and Scleraxis) but not $\operatorname{Sox} 9$, and maintained progenitors into a fibroblastic-like state. However, the addition of BBR to the culture medium at advanced stages of culture promoted chondrogenesis, deduced by the intensification of Alcian blue specific cartilage staining and by the transcriptional up-regulation of $\operatorname{Sox} 9$.

From a clinical point of view, a major question about the suitability of joint cartilage regenerative therapy concerns the nature of the cartilaginous tissue resulting by the treatment. ${ }^{53}$ Our results do not presuppose that the influence of BBR in the micromass assay replicates its pharmacological effects on adult pathologic cartilage but may be of help to uncover effects on degenerating cartilage of interest for therapeutics. The natural outcome of differentiating cartilages in the course of skeletogenesis in vivo and in vitro is a sequential process evolving from prechondrogenic condensation to overt hypertrophy that is followed by osteogenic differentiation. ${ }^{53}$ We observed that ColXa1, Runx2, Ihh, and even Alpl genes, that are considered cartilage prehypertrophic markers, appeared up regulated in the late-stage treated cultures. However, the histological appearance of the cultures together with the twofold increased expression of Scleraxis, and the overexpression of Ccnl (Cyr61) genes, that are associated with the differentiation of progenitors in the contour of the developing cartilage, ${ }^{54}$ indicate that chondrogenic intensification was not fully dependent of a hypertrophic progression of cartilage differentiation. Consistent with this interpretation $\operatorname{Sox} 9$ gene, that is believed to counteract 
cartilage hypertrophy in the growth plate of long bones, ${ }^{55}$ is maintained at high expression levels.

The regulation of Runx 2 in later BBR treatments, merits especial attention. At the beginning of chondrogenic differentiation Runx2 is required to sustain chondrogenesis, while once the cartilage is formed Runx 2 promotes hypertrophy. ${ }^{40}$ Therefore, the differential regulation of Runx 2 in early and late cultures may reflect the stage-dependent opposite influence of BBR on chondrogenesis.

A further task of our study was to identify epigenetic factors as potential targets activated by BBR treatments. We identified Dnmt3b as an epigenetic target potentially implicated in the transcriptional influence of BBR in late cultures and also Dnmt1 and Dnmt3a in early cultures. However, at difference with studies on the anti-tumor influence of $\mathrm{BBR},{ }^{23}$ we detected a significant upregulation, rather than down-regulation, of Dnmt $3 b$. In addition, no transcriptional regulation of other epigenetic markers was appreciated in pro-chondrogenic treatments of BBR. Further studies are required to clarify this question.

\section{Conclusion}

Our study uncovers a pharmacological influence of BBR in the differentiation of the limb skeletal progenitors in vitro. The possibility that these effects were also induced in adult tissues during cartilage degenerative diseases is a tempting hypothesis that may help to design protocols for the treatment of cartilages diseases.

\section{Abbreviations}

BBR, Berberine; DMEM, Dulbecco's Modified Eagle Medium; FBS, fetal bovine serum; PBS, phosphate buffered saline; PI, propidium iodide; qPCR, Real-time quantitative PCR.

\section{Acknowledgments}

We thank Montse Fernandez Calderon, Susana Dawalibi, and Sonia Perez Mantecon for excellent technical assistance. We are grateful to Dr. J. Hurle for comments and suggestions to this study. A Grant (BFU2017-84046-P) from the Spanish Science and Innovation Ministry to $\mathrm{J}$. A.M supported this work.

\section{Disclosure}

The authors report no conflicts of interest in this work.

\section{References}

1. van Wijnen AJ, Westendorf JJ. Epigenetics as a new frontier in orthopedic regenerative medicine and oncology. J Orthop Res. 2019;37(7):1465-1474. doi:10.1002/jor.24305

2. Kumar D, Lassar AB. Fibroblast growth factor maintains chondrogenic potential of limb bud mesenchymal cells by modulating DNMT3A recruitment. Cell Rep. 2014;8(5):1419-1431. doi:10.1016/j.celrep.2014.07.038

3. Christ B, Jacob HJ, Jacob M. Differentiating abilities of avian somatopleural mesoderm. Experientia. 1979;35(10):1376-1378. doi:10.1007/BF01964018

4. Montero JA, Lorda-Diez CI, Sanchez-Fernandez C, Hurle JM. Cell death in the developing vertebrate limb: a locally regulated mechanism contributing to musculoskeletal tissue morphogenesis and differentiation. Dev Dyn. 2020;1-12. doi:10.1002/d vdy. 237

5. Taylor SEB, Li YH, Smeriglio P, Rath M, Wong WH, Bhutani N. Stable 5-hydroxymethylcytosine $(5 \mathrm{hmC})$ acquisition marks gene activation during chondrogenic differentiation. J Bone Miner Res. 2016;31(3):524-534. doi:10.1002/jbmr.2711

6. Sanchez-Fernandez C, Lorda-Diez CI, Hurlé JM, Montero JA. The methylation status of the embryonic limb skeletal progenitors determines their cell fate in chicken. Commun Biol. 2020;3(1):1-12. doi:10.1038/s42003-020-1012-3

7. Ezura Y, Sekiya I, Koga H, Muneta T, Noda M. Methylation status of $\mathrm{CpG}$ islands in the promoter regions of signature genes during chondrogenesis of human synovium-derived mesenchymal stem cells. Arthritis Rheum. 2009;60(5):1416-1426. doi:10.1002/ art. 24472

8. Barter MJ, Bui C, Cheung K, et al. DNA hypomethylation during MSC chondrogenesis occurs predominantly at enhancer regions. Sci Rep. 2020;10(1):1169. doi:10.1038/s41598-020-58093-5

9. Liu CF, Angelozzi M, Haseeb A, Lefebvre V. SOX9 is dispensable for the initiation of epigenetic remodeling and the activation of marker genes at the onset of chondrogenesis. Dev. 2018;145:14. doi:10.1242/dev.164459

10. Webb S, Gabrelow C, Pierce J, Gibb E, Elliott J. Retinoic acid receptor signaling preserves tendon stem cell characteristics and prevents spontaneous differentiation in vitro. Stem Cell Res Ther. 2016;7(1):45. doi:10.1186/s13287-016-0306-3

11. Riasat K, Bardell D, Goljanek-Whysall K, Clegg PD, Peffers MJ. Epigenetic mechanisms in Tendon ageing. Br Med Bull. 2020;135 (1):90-107. doi:10.1093/bmb/ldaa023

12. Zimmermann P, Boeuf S, Dickhut A, Boehmer S, Olek S, Richter W. Correlation of COL10A1 induction during chondrogenesis of mesenchymal stem cells with demethylation of two CpG sites in the COL10A1 promoter. Arthritis Rheum. 2008;58(9):2743-2753. doi:10.1002/art.23736

13. Ramachandran J, Liu Z, Gray RS, Vokes SA. PRMT5 is necessary to form distinct cartilage identities in the knee and long bone. Dev Biol. 2019;456(2):154-163. doi:10.1016/j.ydbio.2019.08.012

14. Kumar A, Ekavali CK, Mukherjee M, Pottabathini R, Dhull DK. Current knowledge and pharmacological profile of berberine: an update. Eur J Pharmacol. 2015;761:288-297. doi:10.1016/j.ejphar.2015.05.068

15. Samadi P, Sarvarian P, Gholipour E, et al. Berberine: a novel therapeutic strategy for cancer. IUBMB Life. 2020;72(10):2065-2079. doi:10.1002/iub. 2350

16. Song D, Hao J, Fan D. Biological properties and clinical applications of berberine. Front Med. 2020;14(5):564-582. doi:10.1007/s11684019-0724-6

17. Zhou Y, Liu S, Peng H, Yu L, He B, Zhao Q. In vivo anti-apoptosis activity of novel berberine-loaded chitosan nanoparticles effectively ameliorates osteoarthritis. Int Immunopharmacol. 2015;28(1):34-43. doi:10.1016/j.intimp.2015.05.014 
18. Lu KY, Lin YC, Lu HT, et al. A novel injectable in situ forming gel based on carboxymethyl hexanoyl chitosan/hyaluronic acid polymer blending for sustained release of berberine. Carbohydr Polym. 2019;206:664-673. doi:10.1016/j.carbpol.2018.11.050

19. Chen Y, Zheng Z, Wang J, et al. Berberine suppresses apoptosis and extracellular matrix (ECM) degradation in nucleus pulposus cells and ameliorates disc degeneration in a rodent model. Int $J$ Biol Sci. 2018;14(6):682-692. doi:10.7150/ijbs.24081

20. Luo R, Liao Z, Song Y, et al. Berberine ameliorates oxidative stress-induced apoptosis by modulating ER stress and autophagy in human nucleus pulposus cells. Life Sci. 2019;228:85-97. doi:10.1016/ j.lfs.2019.04.064

21. Liu X, Wei Y, Bai X, et al. Berberine prevents primary peritoneal adhesion and adhesion reformation by directly inhibiting TIMP-1. Acta Pharm Sin B. 2020;10(5):812-824. doi:10.1016/j.apsb.2020.02.003

22. Shen P, Jiao Y, Miao L, Chen J, Momtazi-Borojeni AA. Immunomodulatory effects of berberine on the inflamed joint reveal new therapeutic targets for rheumatoid arthritis management. $J$ Cell Mol Med. 2020;24(21):12234-12245. doi:10.1111/jcmm.15803

23. Qing Y, Hu H, Liu Y, et al. Berberine induces apoptosis in human multiple myeloma cell line U266 through hypomethylation of p53 promoter. Cell Biol Int. 2014;38(5):563-570. doi:10.1002/cbin.10206

24. Kalaiarasi A, Anusha C, Sankar R, et al. Plant isoquinoline alkaloid berberine exhibits chromatin remodeling by modulation of histone deacetylase to induce growth arrest and apoptosis in the A549 cell line. J Agric Food Chem. 2016;64(50):9542-9550. doi:10.1021/acs.jafc.6b04453

25. Gu C, Yin Z, Nie H, et al. Identification of berberine as a novel drug for the treatment of multiple myeloma via targeting UHRF1. BMC Biol. 2020;18(1):33. doi:10.1186/s12915-020-00766-8

26. Li J, Zou Y, Pei M, Zhang Y, Jiang Y. Berberine inhibits the Warburg effect through TET3/miR-145/HK2 pathways in ovarian cancer cells. J Cancer. 2021;12(1):207-216. doi:10.7150/jca.48896

27. Zhou Y, Tao H, Li Y, et al. Berberine promotes proliferation of sodium nitroprusside-stimulated rat chondrocytes and osteoarthritic rat cartilage via Wnt/ß-catenin pathway. Eur $J$ Pharmacol. 2016;789:109-118. doi:10.1016/j.ejphar.2016.07.027

28. Yamashita M, Inoue $\mathrm{K}$, Saeki $\mathrm{N}$, et al. Uhrfl is indispensable for normal limb growth by regulating chondrocyte differentiation through specific gene expression. Dev. 2018;145:1. doi:10.1242/ dev. 157412

29. Sanchez-Fernandez C, Lorda-Diez CI, García-Porrero JA, Montero JA, Hurlé JM. UHRF genes regulate programmed interdigital tissue regression and chondrogenesis in the embryonic limb. Cell Death Dis. 2019;10(5):1-14. doi:10.1038/s41419-019-1575-4

30. Lorda-Diez CI, Montero JA, Choe S, Garcia-Porrero JA, Hurle JM. Ligand- and stage-dependent divergent functions of BMP signaling in the differentiation of embryonic skeletogenic progenitors in vitro. J Bone Miner Res. 2014;29(3):735-748. doi:10.1002/jbmr.2077

31. Debacq-Chainiaux F, Erusalimsky JD, Campisi J, Toussaint O. Protocols to detect senescence-associated beta-galactosidase (SA$\beta$ gal) activity, a biomarker of senescent cells in culture and in vivo. Nat Protoc. 2009;4(12):1798-1806. doi:10.1038/nprot.2009.191

32. DeLise AM, Stringa E, Woodward WA, Mello MA, Tuan RS. Embryonic limb mesenchyme micromass culture as an in vitro model for chondrogenesis and cartilage maturation. Methods Mol Biol. 2000;137:359-375. doi:10.1385/1-59259-066-7:359

33. Pirosa A, Clark KL, Tan J, et al. Modeling appendicular skeletal cartilage development with modified high-density micromass cultures of adult human bone marrow-derived mesenchymal progenitor cells. Stem Cell Res Ther. 2019;10(1):388. doi:10.1186/s13287-0191505-5

34. Mello MA, Tuan RS. High density micromass cultures of embryonic limb bud mesenchymal cells: an in vitro model of endochondral skeletal development. Vitr Cell Dev Biol. 1999;35(5):262-269. doi:10.1007/s11626-999-0070-0
35. Lorda-Diez CI, Montero JA, Garcia-Porrero JA, Hurle JM. Divergent differentiation of skeletal progenitors into cartilage and Tendon: lessons from the embryonic limb. ACS Chem Biol. 2014;9 (1):72-79. doi:10.1021/cb400713

36. Lorda-Diez CI, Montero JA, Martinez-Cue C, Garcia-Porrero JA, Hurle JM. Transforming growth factors beta coordinate cartilage and tendon differentiation in the developing limb mesenchyme. J Biol Chem. 2009;284(43):29988-29996.

37. Chen H, Ghori-Javed FY, Rashid H, et al. Runx2 regulates endochondral ossification through control of chondrocyte proliferation and differentiation. J Bone Miner Res. 2014;29(12):2653-2665. doi:10.10 02/jbmr.2287

38. Muñoz-Espín D, Serrano M. Cellular senescence: from physiology to pathology. Nat Rev Mol Cell Biol. 2014;15(7):482-496. doi:10.1038/ nrm3823

39. Stott NS, Chuong CM. Dual action of sonic hedgehog on chondrocyte hypertrophy: retrovirus mediated ectopic sonic hedgehog expression in limb bud micromass culture induces novel cartilage nodules that are positive for alkaline phosphatase and type X collagen. $J$ Cell Sci. 1997;110(Pt 2):2691-2701.

40. Enomoto H, Furuichi T, Zanma A, et al. Runx2 deficiency in chondrocytes causes adipogenic changes in vitro. J Cell Sci. 2004;117 (3):417-425. doi:10.1242/jcs.00866

41. Kenny-Mobbs T. Myogenic differentiation in early chick wing mesenchyme in the absence of the brachial somites. J Embryol Exp Morphol. 1985;90:415-436.

42. Montero JA, Lorda-Diez CI, Hurle JM. Confluence of cellular degradation pathways during interdigital tissue remodeling in embryonic tetrapods. Front Cell Dev Biol. 2020;8:1217.

43. Hu Z, Jiao Q, Ding J, et al. Berberine induces dendritic cell apoptosis and has therapeutic potential for rheumatoid arthritis. Arthritis Rheum. 2011;63(4):949-959. doi:10.1002/art.30202

44. Zhou Y, Liu S, Ming J, Li Y, Deng M, He B. Sustained release effects of berberine-loaded chitosan microspheres on in vitro chondrocyte culture. Drug Dev Ind Pharm. 2017;43(10):1703-1714. doi:10.1080/ 03639045.2017.1339076

45. Yue M, Xia Y, Shi C, et al. Berberine ameliorates collagen-induced arthritis in rats by suppressing Th17 cell responses via inducing cortistatin in the gut. FEBS J. 2017;284(17):2786-2801. doi:10.1111/febs.14147

46. Hu P, Chen W, Tang J, Bao J, Wu L. Protective effects of berberine in an experimental rat osteoarthritis model. Phytother Res. 2011;25 (6):878-885. doi:10.1002/ptr.3359

47. Zhao $\mathrm{H}$, Zhang $\mathrm{T}$, Xia $\mathrm{C}$, et al. Berberine ameliorates cartilage degeneration in interleukin-1 $\beta$-stimulated rat chondrocytes and in a rat model of osteoarthritis via Akt signalling. J Cell Mol Med. 2014;18(2):283-292. doi:10.1111/jcmm.12186

48. Wang X, Jiang S, Sun Q. Effects of berberine on human rheumatoid arthritis fibroblast-like synoviocytes. Exp Biol Med (Maywood). 2011;236(7):859-866. doi:10.1258/ebm.2011.010366

49. Ponnusamy L, Kothandan G, Manoharan R. Berberine and Emodin abrogates breast cancer growth and facilitates apoptosis through inactivation of SIK3-induced mTOR and Akt signaling pathway. Biochim Biophys Acta - Mol Basis Dis. 2020;1866(11):165897. doi:10.1016/j.bbadis.2020.165897

50. Gali-Muhtasib H, Hmadi R, Kareh M, Tohme R, Darwiche N. Cell death mechanisms of plant-derived anticancer drugs: beyond apoptosis. Apoptosis. 2015;20(12):1531-1562. doi:10.1007/s10495-015-1169-2

51. Lorda-Diez CI, Garcia-Riart B, Montero JA, Rodriguez-León J, Garcia-Porrero JA, Hurlé JM. Apoptosis during embryonic tissue remodeling is accompanied by cell senescence. Aging (Albany NY). 2015;7(11):974-985. doi:10.18632/aging.100844

52. Lorda-Diez CI, Solis-Mancilla ME, Sanchez-Fernandez C, Garcia-Porrero JA, Hurle JM, Montero JA. Cell senescence, apoptosis and DNA damage cooperate in the remodeling processes accounting for heart morphogenesis. J Anat. 2019;234(6):815-829. doi:10.1111/joa.12972 
53. Hellingman CA, Koevoet W, van Osch GJVM. Can one generate stable hyaline cartilage from adult mesenchymal stem cells? A developmental approach. J Tissue Eng Regen Med. 2012;6(10): e1-e11. doi:10.1002/term.502

54. Lorda-Diez CI, Montero JA, Diaz-Mendoza MJ, Garcia-Porrero JA, Hurle JM. Defining the earliest transcriptional steps of chondrogenic progenitor specification during the formation of the digits in the embryonic limb. PLoS One. 2011;6(9):e24546. doi:10.1371/journal. pone. 0024546
55. Haseeb A, Kc R, Angelozzi M, et al. SOX9 keeps growth plates and articular cartilage healthy by inhibiting chondrocyte dedifferentiation/ osteoblastic redifferentiation. Proc Natl Acad Sci. 2021;118(8): e2019152118. doi:10.1073/pnas.2019152118

\section{Publish your work in this journal}

The Journal of Inflammation Research is an international, peerreviewed open-access journal that welcomes laboratory and clinical findings on the molecular basis, cell biology and pharmacology of inflammation including original research, reviews, symposium reports, hypothesis formation and commentaries on: acute/chronic inflammation; mediators of inflammation; cellular processes; molecular mechanisms; pharmacology and novel anti-inflammatory drugs; clinical conditions involving inflammation. The manuscript management system is completely online and includes a very quick and fair peerreview system. Visit http://www.dovepress.com/testimonials.php to read real quotes from published authors.

Submit your manuscript here: https://www.dovepress.com/journal-of-inflammation-research-journal 